\title{
Imaging Arterial Fibres Using Diffusion Tensor Imaging - Feasibility Study and Preliminary Results
}

\author{
Vittoria Flamini, ${ }^{1}$ Christian Kerskens, ${ }^{2}$ Kevin M. Moerman, ${ }^{3}$ \\ Ciaran K. Simms, ${ }^{3}$ and Caitríona Lally ${ }^{1,3}$ \\ ${ }^{1}$ School of Mechanical and Manufacturing Engineering, Dublin City University, Dublin 9, Ireland \\ ${ }^{2}$ Trinity College Institute for Neuroscience, Trinity College Dublin, Dublin 2, Ireland \\ ${ }^{3}$ Trinity Centre for Bioengineering, School of Engineering, Trinity College Dublin, Dublin 2, Ireland
}

Correspondence should be addressed to Caitríona Lally, triona.lally@dcu.ie

Received 1 May 2009; Revised 13 August 2009; Accepted 21 November 2009

Academic Editor: João Manuel R. S. Tavares

Copyright (C) 2010 Vittoria Flamini et al. This is an open access article distributed under the Creative Commons Attribution License, which permits unrestricted use, distribution, and reproduction in any medium, provided the original work is properly cited.

\begin{abstract}
MR diffusion tensor imaging (DTI) was used to analyze the fibrous structure of aortic tissue. A fresh porcine aorta was imaged at 7T using a spin echo sequence with the following parameters: matrix $128 \times 128$ pixel; slice thickness $0.5 \mathrm{~mm}$; interslice spacing $0.1 \mathrm{~mm}$; number of slices 16; echo time $20.3 \mathrm{~s}$; field of view $28 \mathrm{~mm} \times 28 \mathrm{~mm}$. Eigenvectors from the diffusion tensor images were calculated for the central image slice and the averaged tensors and the eigenvector corresponding to the largest eigenvalue showed two distinct angles corresponding to near $0^{\circ}$ and $180^{\circ}$ to the transverse plane of the aorta. Fibre tractography within the aortic volume imaged confirmed that fibre angles were oriented helically with lead angles of $15 \pm 2.5^{\circ}$ and $175 \pm 2.5^{\circ}$. The findings correspond to current histological and microscopy data on the fibrous structure of aortic tissue, and therefore the eigenvector maps and fibre tractography appear to reflect the alignment of the fibers in the aorta. In view of current efforts to develop noninvasive diagnostic tools for cardiovascular diseases, DTI may offer a technique to assess the structural properties of arterial tissue and hence any changes or degradation in arterial tissue.
\end{abstract}

\section{Introduction}

Cardiovascular diseases are the leading cause of death in the Western world, accounting for nearly half of all the deaths in Europe [1]. The most common arterial diseases are as a result of alterations in the structure of the arterial wall $[2,3]$. Principally, these structural alterations are due to either degeneration of arterial tissue such as in the case of aneurysms [3], or the accumulation of lipids within an artery which can form plaques and stiffen the vessel, as in atherosclerosis [2]. Arterial diseases often progress without symptoms to a point where they sufficiently compromise the circulatory system and subsequently cause a sudden, often fatal event. In fact, aneurysms can dilate an arterial vessel to the point where the vessel tears as a result of the blood pressure, causing a massive haemorrhage [3]. Atherosclerotic plaques can grow within an arterial lumen obstructing blood flow and hence oxygen supply to an organ, causing ischemia
[2]. Ischemia can result in serious damage to vital organs and ultimately can result in myocardial infarction or stroke.

Since arterial diseases may develop in a symptomless way, the best way to diagnose and treat such diseases is by means of preventive medicine and screening $[2,4]$. The optimal screening technique should be noninvasive and capable of detecting early signs of alterations in the arterial structure.

Many hemodynamic studies have investigated the onset of arterial disease in an attempt to provide early indicators of arterial disease that may be detected during diagnostic screening $[4,5]$. They have shown that the arterial wall is an active structure which is subjected to loading and able to respond to environmental changes. In these studies attention has been focussed on alterations in the blood flow pattern in arteries which can create an imbalance in the complex relationship between the forces that regulate the remodelling of the arteries [4, 6-8]. In fact, an injury in the arterial wall or a change in the fluid shear force can trigger an abnormal 
proliferation of the cells, thus causing atherosclerosis. These studies show that the arterial wall is capable of remodelling and it continuously adapts tending towards an optimal balance between stress and strain [4, 7]. In other words, it could be inferred that arterial diseases can be studied by means of solid mechanics and that a disease could be the result of a change in the vessel mechanical properties [9]. This approach could improve the understanding of atherosclerosis and could also be used in determining the aetiology of aneurysms, which, is as yet not completely understood [3].

In order to perform in vivo studies on the solid mechanics of arteries a noninvasive technique that would expose the patient to minimal harm must be used. Noninvasive techniques that are commonly used for the study of arterial diseases include Computed Tomography Angiography (CTA) [10], Magnetic Resonance Angiography (MRA) [11], X-Ray Angiography [12], and colour Doppler Ultrasound [13]. These imaging modalities are limited as they can only image the blood flow and cannot be used to study the mechanics of the arterial wall. They can therefore only provide information on the effect of arterial disease on blood flow and not the underlying cause. Conventional imaging techniques like Computed Tomography (CT) [14] and Magnetic Resonance Imaging (MRI) [15] can be used to image the arterial wall; however they can only provide an anatomical description of a vessel which is insufficient for full mechanical characterization.

In the 1990s, researchers developed an MRI application capable of analysing in vivo the axonal structure of the brain called Diffusion Tensor Imaging (DTI) [16]. DTI is capable of describing the degree of anisotropy of a tissue by analysing the diffusion of water molecules. This motion, which is normally random and hence the same in every direction, that is, isotropic, is altered and constrained in a biological tissue, that is, anisotropic, due to the composition of the underlying microstructure [17]. DTI consists of measuring the diffusion coefficients of water molecules in different directions for each pixel of the image and then creating a diffusion tensor for each pixel [18]. Furthermore, the direction of greatest diffusion, represented by the first eigenvector of the diffusion tensor, can be used to provide information on the fibrous architecture of the tissue, because water molecules will diffuse preferentially along fibres rather than across them [17]. The process of determining the fibre architecture from the diffusion tensor is called fibre tractography [19]. Since the development of DTI and fibre tractography $[16,19]$, these methods have been successfully applied to the brain [20], the heart [21, 22], skeletal muscle [23], cartilage $[24,25]$, and bone [26]. The combination of DTI and fibre tractography has enabled the architecture of the fibrous components of these tissues to be established in vivo.

Arterial tissue can be regarded as a fibre-reinforced material, because different kinds of fibres are present in the arterial wall. The arterial wall can be divided in three layers, each one with its own properties [7, 27]. The inner one is extremely thin and is called the intima. It is composed of endothelium and subendothelium and its role consists of protecting the other layers from plasma lipids and lipoproteins. The middle layer is the media, where both elastic laminae and smooth muscle cells (SMCs) are present. In histological studies reported by Rhodin [27], the elastic laminae are described to be concentrically arranged, while SMCs are reportedly oriented diagonally at small angles, forming a spiral around the vessel. The outer layer is the adventitia, which is dense fibroelastic tissue without smooth muscle cells. Large elastic arteries, such as the aorta, contain high levels of elastin fibres in the media in order that they can withstand the pulsatile pressure waveform produced by the heart whilst more muscular arteries contain higher levels of smooth muscle cells and collagen and lower levels of elastin $[7,27]$. The quantity and distribution of fibres within the arterial wall and their quality is therefore a direct measure of the mechanical strength and the health of arterial tissue [9].

In this study the aim was to assess the applicability of DTI for determining the fibre structure of arterial tissue. In particular, DTI was evaluated to establish if it could determine the helical and near circumferential arrangement of fibres within the aorta that has been extensively reported to be present within arterial tissue.

\section{Materials and Methods}

A porcine aorta was harvested from a six-month-old pig of Irish breed. The thoracic-abdominal section of the aorta was cut from the complete aorta. The vessel was $122 \mathrm{~mm}$ long and had a thickness of $2 \mathrm{~mm}$ in the proximal section and $1.5 \mathrm{~mm}$ in the distal one. The external connective tissue was removed from the aorta, and the vessel was placed in a custom designed cylindrical chamber filled with water. The chamber had dimensions of $32 \mathrm{~mm}$ diameter and $200 \mathrm{~mm}$ length. The chamber was designed to fit a circular polarised whole body Radio Frequency coil for a 7T Biospec (Bruker Biospin, Germany) Magnetic Resonance Imaging (MRI) scanner. The sample was scanned within 24 hours of slaughter according to the following Diffusion Tensor Imaging (DTI) acquisition protocol: spin echo sequence; matrix $128 \times 128$ pixel; slice thickness $0.5 \mathrm{~mm}$; interslice spacing $0.1 \mathrm{~mm}$; number of slices 16 ; echo time $20.3 \mathrm{~s}$; field of view $28 \mathrm{~mm} \times 28 \mathrm{~mm}$.

Diffusion Tensor Imaging is characterised by the application of a diffusion sensitivity gradient over at least six noncoplanar directions and by the application of a particular $b$ value, where the $b$ value is a measure of the sensitivity to diffusion, defined as follows:

$$
\frac{S}{S_{0}}=\exp (-b D),
$$

where $S$ is the signal of the image analysed with an encoding gradient, $S_{0}$ is the signal of a reference image (i.e., one taken with a null gradient), and $D$ is the diffusion tensor [17]. The $b$ value influences the signal-to-noise ratio and also describes the impact that the diffusion has on the image: when the $b$ value increases, water molecular diffusion increases and therefore the signal of the image, $S$, diminishes along the direction of the gradient and the signal-to-noise 


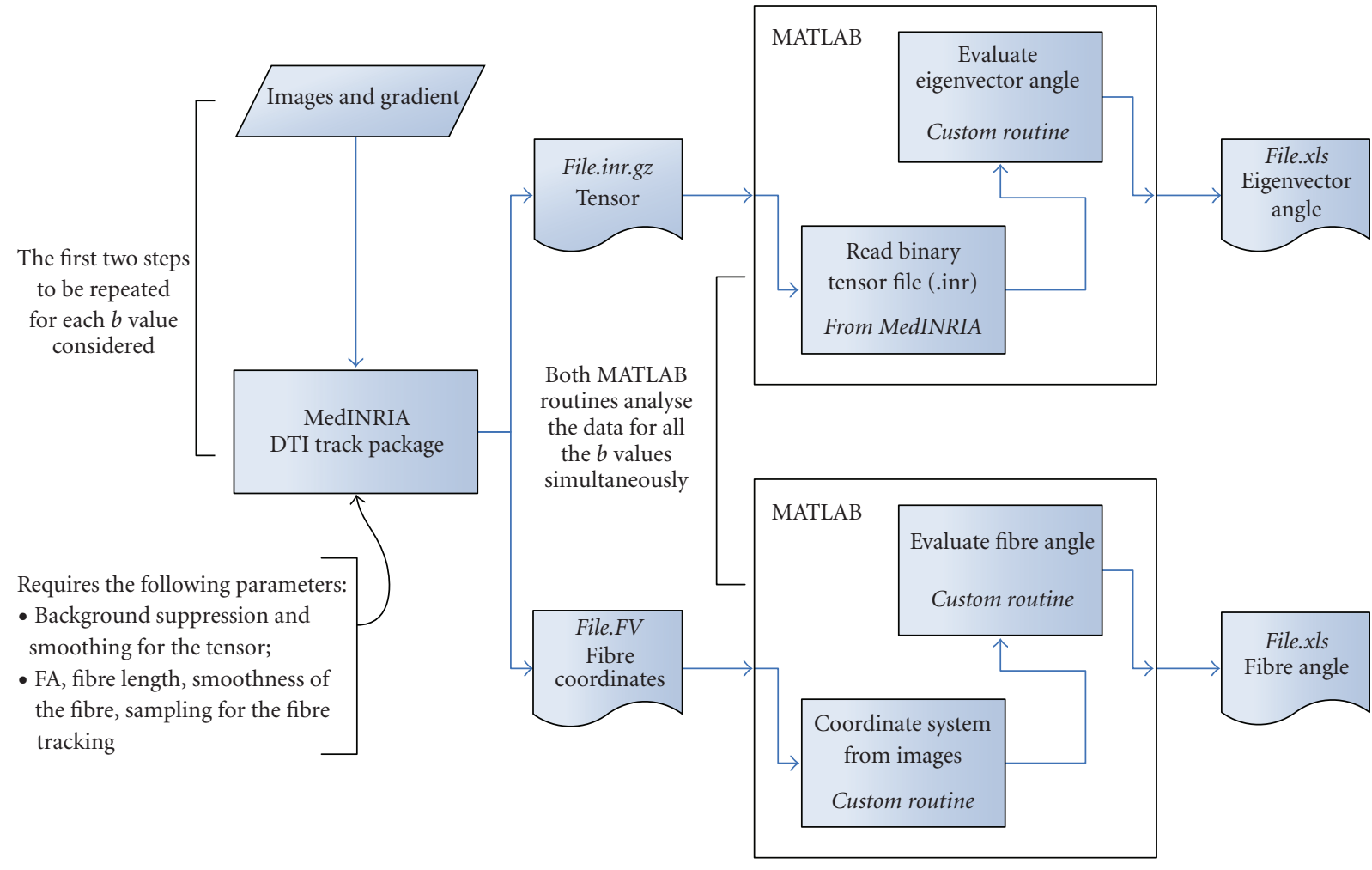

FIGURE 1: Flow chart indicating the various stages in the image postprocessing sequence.

ratio decreases $[28,29]$. In contrast, for low $b$ values the signal-to-noise ratio can be high but diffusion of water molecules along fibres is so low that fibre tracking may be impeded. The $b$ value and the gradient are connected: the $b$ value is proportional to gradient parameters such as amplitude, duration, and time spacing and the most suitable value depends on the tissue type being imaged $[29,30]$. Therefore an optimal $b$ value for arterial tissue had to be determined. In this study the gradient was applied over six diffusion directions and scans were repeated for six different $b$ values; in particular the values analysed were: $200,400,600,800,1200$, and $1600 \mathrm{~s} / \mathrm{mm}^{2}$. Five repetitions of each measurement were taken and then averaged using a custom routine implemented in MATLAB. Averaging the measurements over five repetitions ensured that the results were more robust; however, measurements obtained from only one repetition where only the central slice of the image was considered and where all the slices were considered showed very little deviation from the averaged results of the five repetitions; see Tables 1 and 2, respectively.

By analysing the images taken for different diffusion directions for each pixel it is possible to derive a tensor that contains the information regarding the local diffusivity. Moreover, eigenvalues and eigenvectors can be extrapolated from each diffusion tensor [18]. Diffusion eigenvalues are important for the determination of a parameter called fractional anisotropy (FA) [30]. The FA is an index of the anisotropy of diffusion in the tissue and ranges between 0 and 1 , with 0 being isotropy and 1 being complete anisotropy. The fractional anisotropy is defined according to (2), where $D$ is the diffusion tensor, $\lambda_{1}, \lambda_{2}, \lambda_{3}$ are its eigenvalues, and $\operatorname{tr}(D)$ is the trace of $D[30]$ :

$$
\begin{aligned}
& \mathrm{FA}=\sqrt{\frac{3}{2}} \sqrt{\frac{\left(\lambda_{1}-\bar{D}\right)^{2}+\left(\lambda_{2}-\bar{D}\right)^{2}+\left(\lambda_{3}-\bar{D}\right)^{2}}{\lambda_{1}^{2}+\lambda_{2}^{2}+\lambda_{3}^{2}}}, \\
& \bar{D}=\frac{\operatorname{tr}(D)}{3} \text {. }
\end{aligned}
$$

In addition, diffusion eigenvectors are important for the determination of fibres patterns; the first eigenvector (i.e., the vector corresponding to the largest eigenvalue of the tensor) represents the direction of maximal diffusion and therefore it represents the predominant fibre direction $[17,19]$. Fibre tractography can be defined as the pixelwise interpolation of the directions of the first eigenvector. Different interpolation algorithms are available, and in this study the algorithm implemented for the DTI fibre analysis was that available in the software MedINRIA (Sophia Antipolis, France). This software was chosen because it is optimised for DTI on clinical datasets. In fact, in order to reduce the noise which is common in these kinds of acquisitions, MedINRIA applies a maximum likelihood strategy. The estimation of the tensor, together with the use of Log-Euclidean metrics for tensor processing, improves the quality of the fibres reconstructed, which are tracked by using a streamline algorithm [31]. Using MedINRIA the diffusion tensor for each $b$ value was evaluated and the fibre tractography was performed. In order 
TABLE 1: Evaluation of the difference in the eigenvector angles between each repetition and the average over all the repetitions for the central slice of the image.

\begin{tabular}{|c|c|c|c|c|c|}
\hline & & \multicolumn{2}{|c|}{ Angles between $0^{\circ}-90^{\circ}$} & \multicolumn{2}{|c|}{ Angles between $90^{\circ}-180^{\circ}$} \\
\hline & & $\begin{array}{l}\text { Most prevalent } \\
\text { angle range }\left({ }^{\circ}\right)\end{array}$ & $\begin{array}{c}\% \text { of } \\
\text { occurrence }\end{array}$ & $\begin{array}{l}\text { Most prevalent } \\
\text { angle range }\left({ }^{\circ}\right)\end{array}$ & $\begin{array}{c}\% \text { of } \\
\text { occurrence }\end{array}$ \\
\hline \multirow{6}{*}{ Repetition n.1 } & $b 1$ & $5 \pm 2.5$ & 7.29 & $175 \pm 2.5$ & 4.19 \\
\hline & $b 2$ & $5 \pm 2.5$ & 9.12 & $175 \pm 2.5$ & 6.50 \\
\hline & $b 3$ & $5 \pm 2.5$ & 12.35 & $175 \pm 2.5$ & 8.42 \\
\hline & $b 4$ & $5 \pm 2.5$ & 13.49 & $175 \pm 2.5$ & 11.00 \\
\hline & $b 5$ & $5 \pm 2.5$ & 15.76 & $175 \pm 2.5$ & 10.87 \\
\hline & $b 6$ & $5 \pm 2.5$ & 17.50 & $175 \pm 2.5$ & 10.87 \\
\hline \multirow{6}{*}{ Repetition n.2 } & $b 1$ & $5 \pm 2.5$ & 8.64 & $175 \pm 2.5$ & 5.94 \\
\hline & $b 2$ & $5 \pm 2.5$ & 10.74 & $175 \pm 2.5$ & 8.77 \\
\hline & $b 3$ & $5 \pm 2.5$ & 13.23 & $175 \pm 2.5$ & 12.31 \\
\hline & $b 4$ & $5 \pm 2.5$ & 15.58 & $175 \pm 2.5$ & 12.13 \\
\hline & $b 5$ & $5 \pm 2.5$ & 17.29 & $175 \pm 2.5$ & 13.66 \\
\hline & $b 6$ & $5 \pm 2.5$ & 18.33 & $175 \pm 2.5$ & 15.28 \\
\hline \multirow{6}{*}{ Repetition n.3 } & $b 1$ & $5 \pm 2.5$ & 9.04 & $175 \pm 2.5$ & 6.90 \\
\hline & $b 2$ & $5 \pm 2.5$ & 14.14 & $175 \pm 2.5$ & 10.13 \\
\hline & $b 3$ & $5 \pm 2.5$ & 13.71 & $175 \pm 2.5$ & 11.39 \\
\hline & $b 4$ & $5 \pm 2.5$ & 17.63 & $175 \pm 2.5$ & 11.39 \\
\hline & $b 5$ & $5 \pm 2.5$ & 19.21 & $175 \pm 2.5$ & 10.43 \\
\hline & $b 6$ & $5 \pm 2.5$ & 19.07 & $175 \pm 2.5$ & 14.01 \\
\hline \multirow{6}{*}{ Repetition n.4 } & $b 1$ & $5 \pm 2.5$ & 8.77 & $175 \pm 2.5$ & 5.46 \\
\hline & $b 2$ & $5 \pm 2.5$ & 13.18 & $175 \pm 2.5$ & 8.90 \\
\hline & $b 3$ & $5 \pm 2.5$ & 13.05 & $175 \pm 2.5$ & 10.91 \\
\hline & $b 4$ & $5 \pm 2.5$ & 14.80 & $175 \pm 2.5$ & 11.65 \\
\hline & $b 5$ & $5 \pm 2.5$ & 18.07 & $175 \pm 2.5$ & 12.75 \\
\hline & $b 6$ & $5 \pm 2.5$ & 16.89 & $175 \pm 2.5$ & 11.48 \\
\hline \multirow{6}{*}{ Repetition n.5 } & $b 1$ & $5 \pm 2.5$ & 8.47 & $175 \pm 2.5$ & 6.11 \\
\hline & $b 2$ & $5 \pm 2.5$ & 10.30 & $175 \pm 2.5$ & 8.77 \\
\hline & $b 3$ & $5 \pm 2.5$ & 14.80 & $175 \pm 2.5$ & 11.48 \\
\hline & $b 4$ & $5 \pm 2.5$ & 16.06 & $175 \pm 2.5$ & 12.13 \\
\hline & $b 5$ & $5 \pm 2.5$ & 19.82 & $175 \pm 2.5$ & 13.09 \\
\hline & $b 6$ & $5 \pm 2.5$ & 19.82 & $175 \pm 2.5$ & 13.09 \\
\hline \multirow{6}{*}{ Averaged repetitions } & $b 1$ & $20 \pm 2.5$ & 9.15 & $160 \pm 2.5$ & 8.77 \\
\hline & $b 2$ & $5 \pm 2.5$ & 11.41 & $175 \pm 2.5$ & 10.88 \\
\hline & $b 3$ & $5 \pm 2.5$ & 15.28 & $175 \pm 2.5$ & 12.39 \\
\hline & $b 4$ & $5 \pm 2.5$ & 17.92 & $175 \pm 2.5$ & 11.62 \\
\hline & $b 5$ & $5 \pm 2.5$ & 19.01 & $175 \pm 2.5$ & 15.14 \\
\hline & $b 6$ & $5 \pm 2.5$ & 18.56 & $175 \pm 2.5$ & 15.14 \\
\hline
\end{tabular}

to proceed with the fibre tractography a region of interest (ROI) was manually defined that corresponded to the area between the external and internal boundary of the aorta, as delineated from the central image slice of the aorta. The software then tracked all the fibres passing through that ROI. The fibre tractography parameters were determined through previous DTI empirical measurements on aortic tissue and these parameters include the FA, the sampling pixel number, the minimum fibre length in $\mathrm{mm}$ and the smoothing interpolation of the fibres. These parameters were defined as follows: the FA was set to 0.2 , the value for which no fibres were tracked in the water; the sampling pixel number was set to 3, the number of pixels used to determine the initial fibre vector direction; the minimum fibre length was set to $10 \mathrm{~mm}$; and the smoothing of the interpolated fibre was set to $20 \%$ [32]; see the appendix for more details on the process used to determine these parameters.

Subsequently, in MATLAB (Natick, MA, USA) two custom routines were implemented, one for the analysis of the tensor and one for the analysis of the orientation of fibres; see Figure 1. The tensor analysis consisted of the extrapolation of the first eigenvector from the tensor, and 
TABLE 2: Evaluation of the difference in the eigenvector angles between each repetition and the average over all of the repetitions. In this case the measurement is averaged over all of the slices of the volume.

\begin{tabular}{|c|c|c|c|c|c|}
\hline & & \multicolumn{2}{|c|}{ Angles between $0^{\circ}-90^{\circ}$} & \multicolumn{2}{|c|}{ Angles between $90^{\circ}-180^{\circ}$} \\
\hline & & $\begin{array}{l}\text { Most prevalent } \\
\text { angle range }\left(^{\circ}\right)\end{array}$ & $\begin{array}{c}\% \text { of } \\
\text { occurrence }\end{array}$ & $\begin{array}{l}\text { Most prevalent } \\
\text { angle range }\left(^{\circ}\right)\end{array}$ & $\begin{array}{c}\% \text { of } \\
\text { occurrence }\end{array}$ \\
\hline \multirow{6}{*}{ Repetition n.1 } & $b 1$ & $5 \pm 2.5$ & 7.09 & $175 \pm 2.5$ & 4.36 \\
\hline & $b 2$ & $5 \pm 2.5$ & 9.76 & $175 \pm 2.5$ & 6.72 \\
\hline & $b 3$ & $5 \pm 2.5$ & 11.33 & $175 \pm 2.5$ & 8.42 \\
\hline & $b 4$ & $5 \pm 2.5$ & 12.55 & $175 \pm 2.5$ & 10.06 \\
\hline & $b 5$ & $5 \pm 2.5$ & 15.08 & $175 \pm 2.5$ & 10.72 \\
\hline & $b 6$ & $5 \pm 2.5$ & 14.99 & $175 \pm 2.5$ & 10.43 \\
\hline \multirow{6}{*}{ Repetition n.2 } & $b 1$ & $5 \pm 2.5$ & 8.69 & $175 \pm 2.5$ & 5.50 \\
\hline & $b 2$ & $5 \pm 2.5$ & 10.85 & $175 \pm 2.5$ & 8.34 \\
\hline & $b 3$ & $5 \pm 2.5$ & 12.70 & $175 \pm 2.5$ & 10.13 \\
\hline & $b 4$ & $5 \pm 2.5$ & 14.21 & $175 \pm 2.5$ & 11.15 \\
\hline & $b 5$ & $5 \pm 2.5$ & 16.28 & $175 \pm 2.5$ & 11.37 \\
\hline & $b 6$ & $5 \pm 2.5$ & 15.63 & $175 \pm 2.5$ & 12.48 \\
\hline \multirow{6}{*}{ Repetition n.3 } & $b 1$ & $5 \pm 2.5$ & 8.18 & $175 \pm 2.5$ & 6.35 \\
\hline & $b 2$ & $5 \pm 2.5$ & 11.59 & $175 \pm 2.5$ & 9.21 \\
\hline & $b 3$ & $5 \pm 2.5$ & 12.96 & $175 \pm 2.5$ & 10.72 \\
\hline & $b 4$ & $5 \pm 2.5$ & 15.93 & $175 \pm 2.5$ & 11.24 \\
\hline & $b 5$ & $5 \pm 2.5$ & 16.19 & $175 \pm 2.5$ & 11.48 \\
\hline & $b 6$ & $5 \pm 2.5$ & 17.59 & $175 \pm 2.5$ & 12.72 \\
\hline \multirow{6}{*}{ Repetition n.4 } & $b 1$ & $5 \pm 2.5$ & 8.12 & $175 \pm 2.5$ & 5.52 \\
\hline & $b 2$ & $5 \pm 2.5$ & 11.48 & $175 \pm 2.5$ & 9.54 \\
\hline & $b 3$ & $5 \pm 2.5$ & 12.85 & $175 \pm 2.5$ & 10.32 \\
\hline & $b 4$ & $5 \pm 2.5$ & 14.45 & $175 \pm 2.5$ & 11.04 \\
\hline & $b 5$ & $5 \pm 2.5$ & 16.17 & $175 \pm 2.5$ & 11.11 \\
\hline & $b 6$ & $5 \pm 2.5$ & 16.80 & $175 \pm 2.5$ & 11.26 \\
\hline \multirow{6}{*}{ Repetition n.5 } & $b 1$ & $5 \pm 2.5$ & 7.42 & $175 \pm 2.5$ & 5.63 \\
\hline & $b 2$ & $5 \pm 2.5$ & 11.46 & $175 \pm 2.5$ & 8.36 \\
\hline & $b 3$ & $5 \pm 2.5$ & 12.44 & $175 \pm 2.5$ & 10.10 \\
\hline & $b 4$ & $5 \pm 2.5$ & 13.29 & $175 \pm 2.5$ & 12.00 \\
\hline & $b 5$ & $5 \pm 2.5$ & 16.24 & $175 \pm 2.5$ & 12.31 \\
\hline & $b 6$ & $5 \pm 2.5$ & 16.24 & $175 \pm 2.5$ & 12.31 \\
\hline \multirow{6}{*}{ Averaged repetition } & $b 1$ & $20 \pm 2.5$ & 9.14 & $175 \pm 2.5$ & 7.45 \\
\hline & $b 2$ & $5 \pm 2.5$ & 10.28 & $175 \pm 2.5$ & 9.35 \\
\hline & $b 3$ & $5 \pm 2.5$ & 12.57 & $175 \pm 2.5$ & 10.14 \\
\hline & $b 4$ & $5 \pm 2.5$ & 14.65 & $175 \pm 2.5$ & 10.48 \\
\hline & $b 5$ & $5 \pm 2.5$ & 13.45 & $175 \pm 2.5$ & 13.20 \\
\hline & $b 6$ & $5 \pm 2.5$ & 14.03 & $175 \pm 2.5$ & 13.31 \\
\hline
\end{tabular}

the determination of the angle it formed with the $x-y$ plane, as illustrated in Figure 2(a). This was conducted on a single slice of the image (the central one). In order to study the consistency of the results over the length of the sample, the average of the tensor over all the slices was considered, and the angle of the eigenvector calculated. In both cases the study was focused on the ROI defined in MedINRIA.

The fibre distribution was analysed in another routine that assumed each fibre to be a portion of a helix. Consequently, the fibres could be represented by the following set of equations which are the general equations for a helix [33]:

$$
\begin{gathered}
x=R \cos (t), \\
y=R \sin (t), \\
z=c t,
\end{gathered}
$$

where $t$ is the angle with the $x$ axis, $R$ is the radius and $c$ is the lead. From these equations the definition of the helix angle can be derived and used to define the lead fibre angle, that is, the angle shown in Figure 2(b), as follows:

$$
\tan (\theta)=\frac{c}{R}
$$




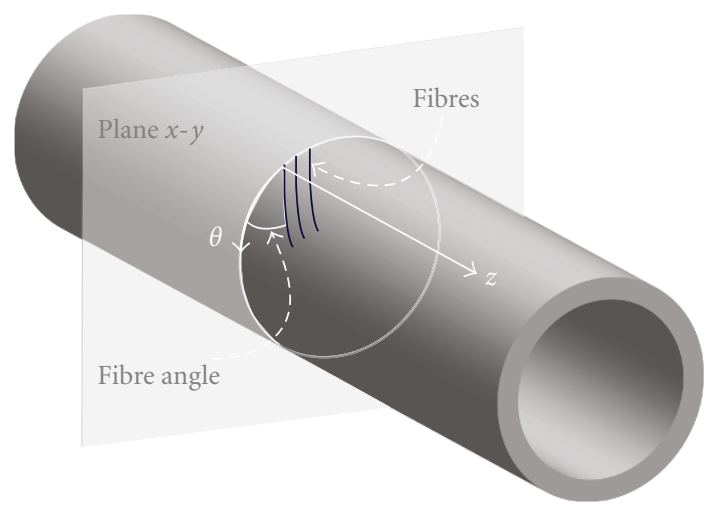

(a)

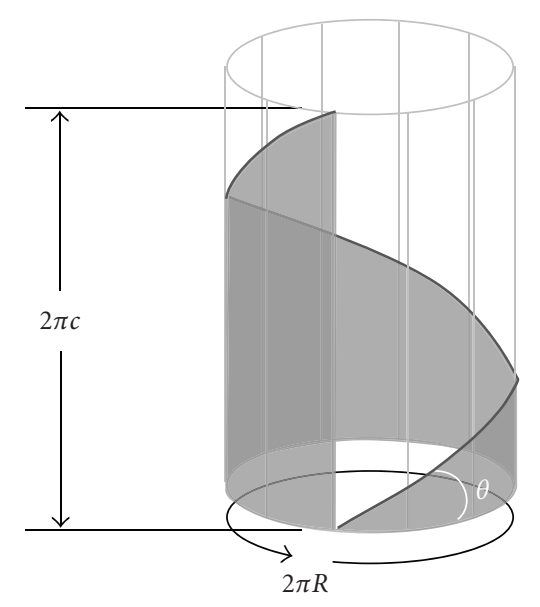

(b)

Figure 2: (a) Convention for the lead fibre angles calculated in this study; (b) definition of the fibre angle.

In order to apply these equations the fibres' coordinates, which were stored in an ASCII coded text file, needed to be converted from the image reference system to cylindrical coordinates, and therefore a centre had to be determined. Therefore, the ROI mask was used to determine the centre of mass of the aortic section and this was taken as the origin of the reference cylindrical coordinate system. Once the coordinates were converted, (4) was applied and the resultant fibre angle distribution was computed. For each fibre, the fibre angle was evaluated for each point of the fibre and then the median was taken. Test helices were created in MATLAB for the purpose of testing this routine. The helices had known angles $\left(30^{\circ}, 45^{\circ}\right.$, and $\left.-30^{\circ}\right)$, and the routine described above was successful in determining their lead angles.

\section{Results}

The process of determining the fibrous structure of the aortic tissue is illustrated in Figure 3, where all of the steps in the imaging and postprocessing procedure are shown. Firstly, the anatomical image resulting from the scan is used to determine the ROI; see Figures 3(a) and 3(b). Secondly, the diffusion tensor is analysed in MATLAB and the angle between the first eigenvector and the $x-y$ plane determined and mapped onto the ROI; see Figures 3(c) and 3(d). From both of these images it can be seen that the region of the aorta in the image is still recognisable using the tensor map. Finally, the tensor is analysed using MedINRIA and the fibres tracked through the ROI of the aorta; see Figures 3(e) and 3(f). From these images it can be seen that the fibres plotted are distributed throughout the thickness of the aorta and that they are predominantly oriented circumferentially within the $x-y$ plane of the aorta.

The results for the tensor orientation were analysed for different $b$ values to determine the influence of the $b$ value on the tensor angles obtained. For the tensor representing the central slice and the averaged tensor, the angle between the first eigenvector and the $x-y$ plane had greater variability for small $b$ values and became increasingly more consistent at higher $b$ values; see Figures 4 and 5. Two dominant eigenvector angles, close to $0^{\circ}$ and $180^{\circ}$, are evident for the analysis of the tensors of the central slice image for all $b$ values (Figure 4 ), whilst three, close to $0^{\circ}, 90^{\circ}$ and $180^{\circ}$, are present in the averaged images (Figure 5). However, by using the parameters defined above to carry out the fibre tractography such that the fibre angles were tracked, two dominant fibre angles were found between $15^{\circ} \pm 2.5^{\circ}$ and $175^{\circ} \pm 2.5^{\circ}$, respectively (Figures 6 and 7 ). These angles were found to be independent of the $b$ value applied during the imaging sequence. In the fibre tractography plots (Figures 6 and 7), the fibre angle distribution is evaluated over bands of $5^{\circ}$, and centred in the middle of each band.

\section{Discussion and Conclusions}

The arterial wall constitutes a highly organized tissue which must withstand a complex network of forces acting on it, as shown by Burton [6] and Peterson et al. [34]. The organisation of the tissue is therefore of utmost importance, as it has to offer distensibility and resistance [7]. The arterial tissue mechanical properties are derived from its microstructure which is constituted by collagen, elastin fibres, SMCs, and ground substances [27]. The fibrous components reinforce the structure and their distribution generally corresponds to the direction of maximum stress $[6,7]$. The orientation of arterial fibrous components has been studied with many different techniques including histology [27], scanning electron microscopy (SEM) [35], confocal electron microscopy [36], and confocal laser scanning microscopy [37]. All of these techniques were consistent in finding that arterial tissue fibres are woven according to a helical pattern with a small pitch. In particular, in the study from O'Connell et al. [37], where the three-dimensional architecture of arterial fibres was reconstructed by means of microscopy, they demonstrated that all three fibrous constituents of the artery (i.e., collagen, elastin fibres, and SMCs) are aligned predominantly in the circumferential direction and in particular approximately $\pm 10^{\circ}$ from the circumferential direction. The results presented in the current study are in accordance with this result. Firstly, by looking at Tables 1 and 2 it can be seen that in every repetition (as well as in the averaged 


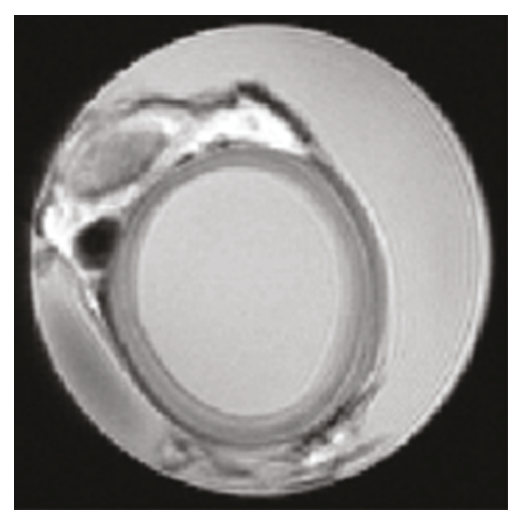

(a)

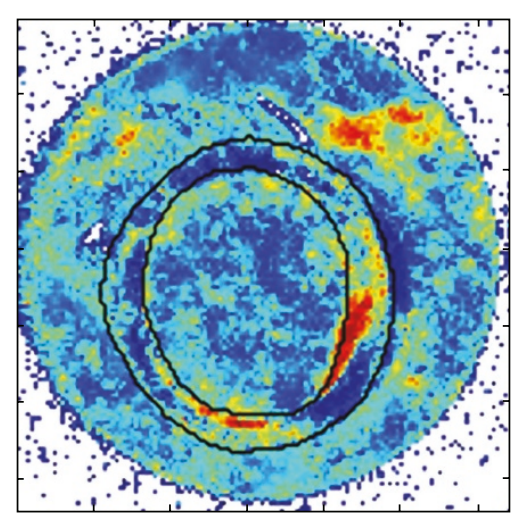

(d)

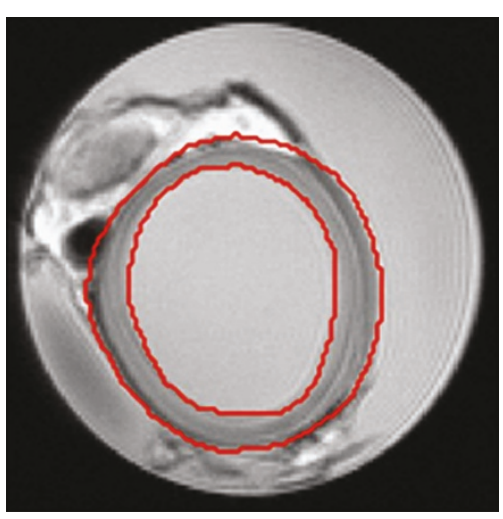

(b)

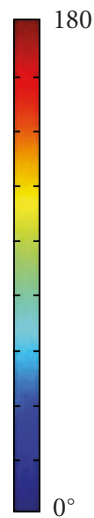

$0^{\circ}$

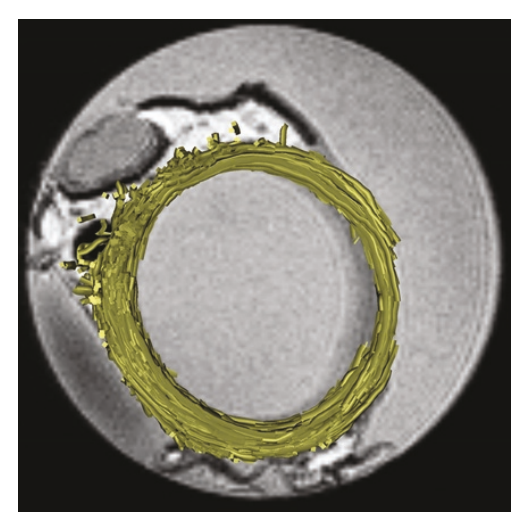

(e)

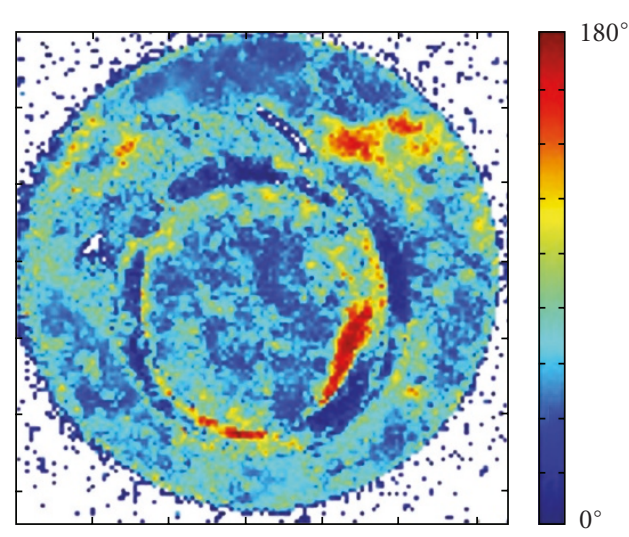

(c)

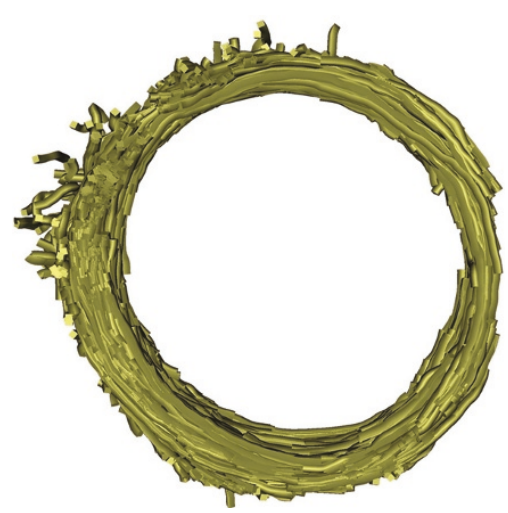

(f)

FIGURE 3: Steps in the DTI procedure and image postprocessing; (a) MRI anatomical scan, (b) the ROI of the aorta, (c) a map of the angle of the first eigenvector with the $x-y$ plane, (d) a map of the angle of the first eigenvector with the $x-y$ plane with the ROI clearly identified, (e) the results of the tractography process with the fibres superimposed on the reference image, and (f) the aortic fibres within the ROI alone.

repetitions) the eigenvector angle is predominantly oriented in the range of $5 \pm 2.5^{\circ}$ and $175 \pm 2.5^{\circ}$. The tensor maps, where the angle of the first eigenvector with the $x-y$ plane is mapped, also show that the main diffusion direction has a small angle. In particular, by looking at the map for a single slice, it is clear to see that only the angular extremes, $0^{\circ}$ and $180^{\circ}$, are evident on the contour map of the artery (Figure 4). This trend was seen in all individual slices where the eigenvector of the diffusion tensor was determined; however, when considering the overall sample, as in Figure 4, areas with eigenvectors at $90^{\circ}$ to the $x-y$ plane are also present. By comparing the maps of the pixelwise eigenvectors for individual slices (central slices are shown in Figure 4) to that of the averaged tensor (Figure 5), it appears that some changes in the diffusion direction occur in parts of the vessel such that pixels with $0^{\circ}$ and $180^{\circ}$ eigenvector angles in different slices when averaged result in an angle of $90^{\circ}$. Therefore, analysis of the averaged tensor gives an indication of changes in the diffusion along the length of the vessel whilst individual slices give information on the local diffusion and may be indicators of fibre directions in specific regions of the vessel.

To establish fibre directions more conclusively, fibre tractography needs to be performed and the fibre tractography on the diffusion tensors in the current study identified dominant fibre angles of $15 \pm 2.5^{\circ}$ and $175 \pm 2.5^{\circ}$, as seen in Figures 6 and 7. This is consistent with the fibre direction reported in the literature for arterial tissue by O'Connell et al. [37]. This result is also in agreement with the eigenvector angles obtained directly from the diffusion tensor. Differences between the eigenvector angles and the fibre angles are to be expected due to the fact that these can be regarded as two different entities. In fact, even though the determination of the fibres is based on eigenvector angles, it is the three-dimensional eigenvector arrangement that dictates the fibre together with the constraints imposed by the tractography algorithm.

All of these results support the use of DTI as a means of obtaining a reliable description of the natural fibre orientation of arterial tissue in a noninvasive way; whereas techniques such as histology and microscopy need the tissue to be harvested and fixed. Harvesting the vessel, whilst clearly invasive, also has implications for the structural properties since that it removes any in situ longitudinal or circumferential prestretches. Moreover, with most of these techniques only small bi-dimensional portions of the arterial wall can be analysed, while with DTI it is possible to obtain the global, three-dimensional, fibre orientations. 


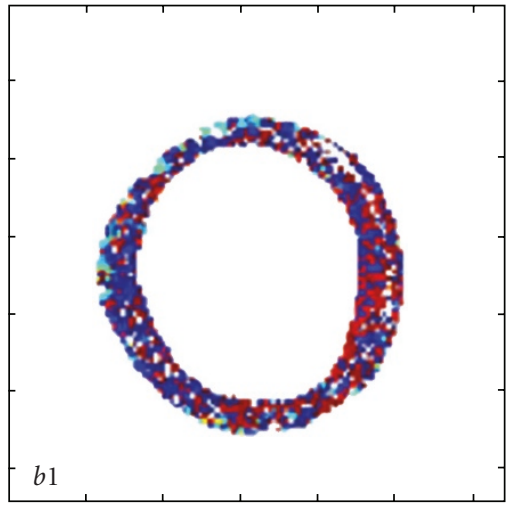

(a)

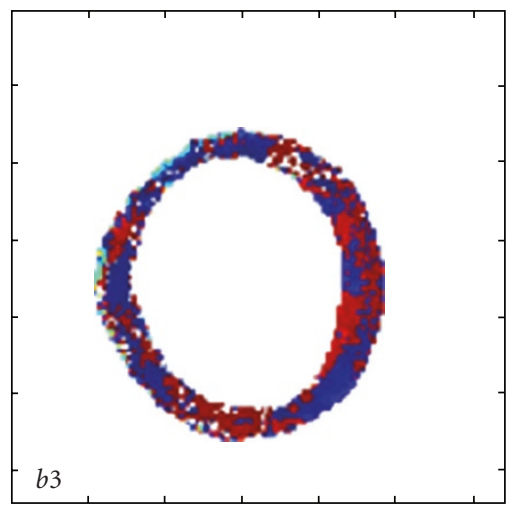

(c)

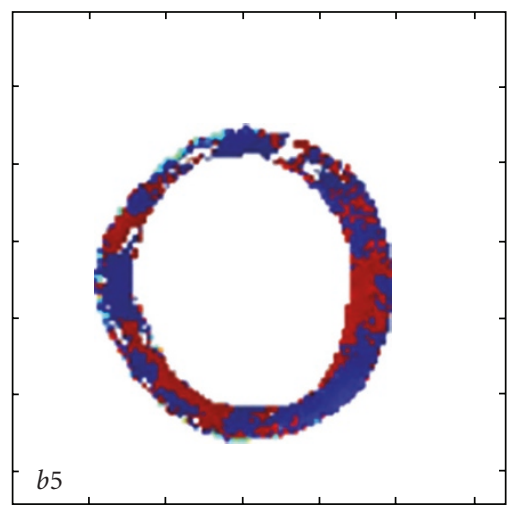

(e)
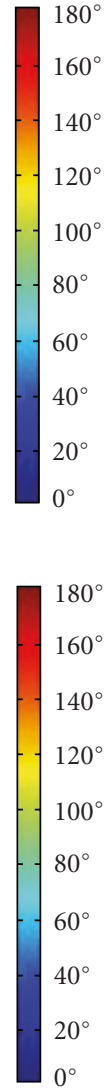

$180^{\circ}$
$160^{\circ}$
$140^{\circ}$
$120^{\circ}$
$100^{\circ}$
$80^{\circ}$
$60^{\circ}$
$40^{\circ}$
$20^{\circ}$
$0^{\circ}$

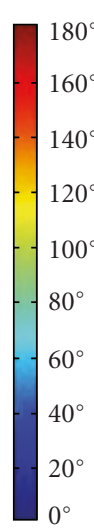

$0^{\circ}$

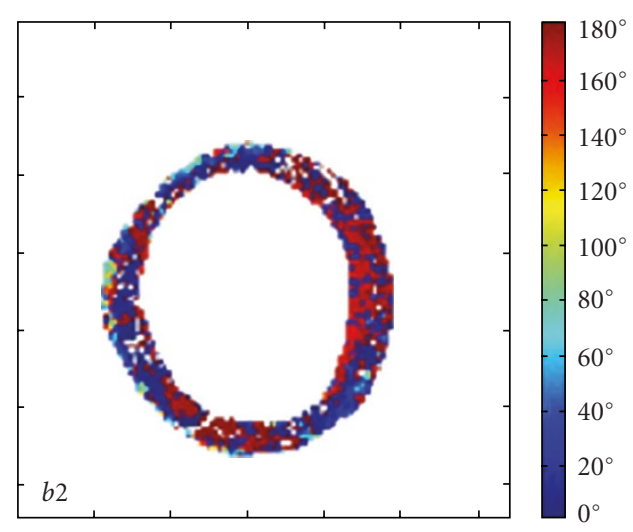

(b)

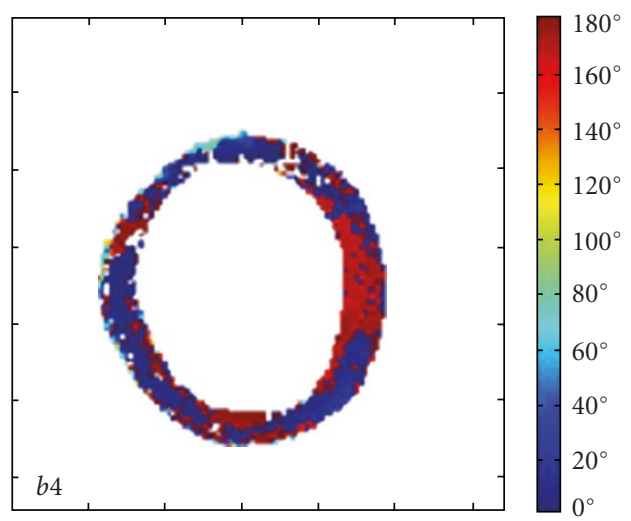

(d)

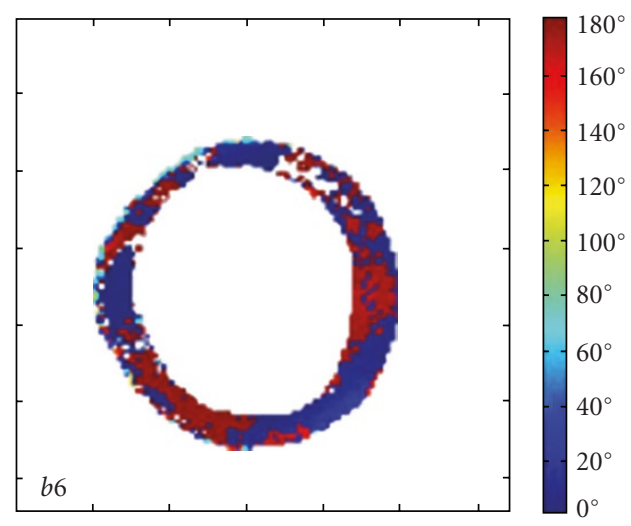

(f)

FIGURE 4: Contour maps of the angle between the first eigenvector and the $x-y$ plane for the central slices of the image data sets for the different $b$ values.

Another interesting feature of this study is the determination of the most appropriate $b$ value for the analysis of the fibrous orientation within the arterial wall. The optimal $b$ value in DTI is dependant on the tissue being studied; for example, a value of $1000 \mathrm{~s} / \mathrm{mm}^{2}$ has been reported for cartilage [24], whilst $400 \mathrm{~s} / \mathrm{mm}^{2}$ has been used for the medial nerve in the human wrist $[28,38]$, and values between 500 and $800 \mathrm{~s} / \mathrm{mm}^{2}$ for the myocardium $[39,40]$. The $b$ value appears to be connected with the composition of the tissue studied and therefore can be used for the diagnosis of diseases that alter such composition [41, 42].

To the best of the authors' knowledge a suitable $b$ value for DTI of arteries has not been reported to date and therefore a range of increasing $b$ values were used in this feasibility study. To find the optimal $b$ value the information in each image set for this range of $b$ values had to be analysed, in particular the amount of significant data obtained in each image had to be quantified. For each $b$ value the 


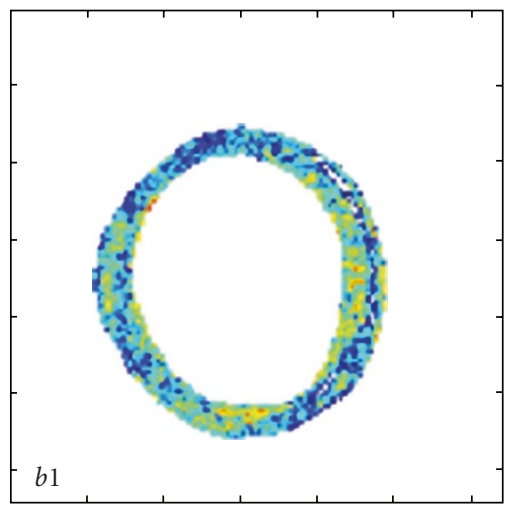

(a)

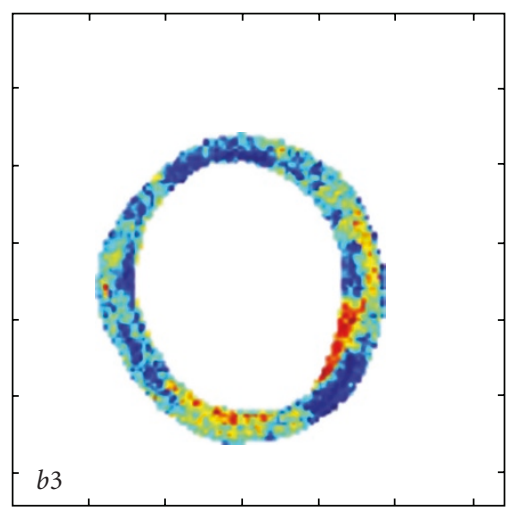

(c)

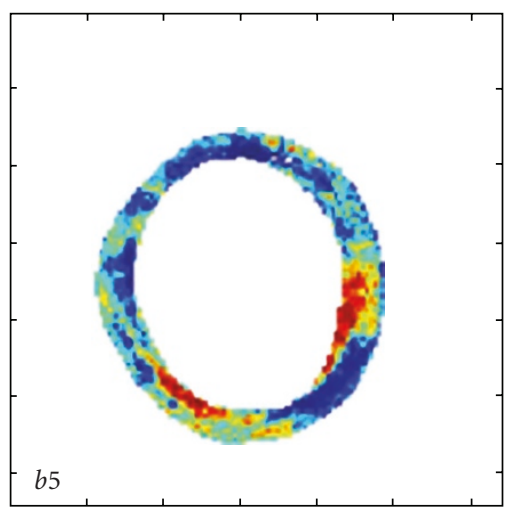

(e)
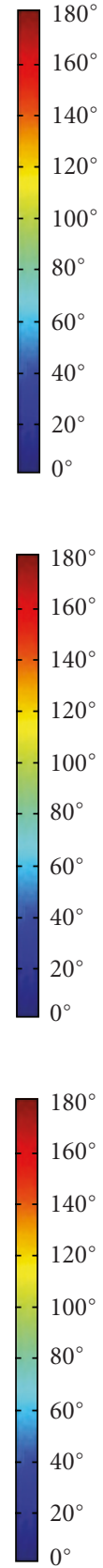

$180^{\circ}$
$160^{\circ}$
$140^{\circ}$
$120^{\circ}$
$100^{\circ}$
$80^{\circ}$
$60^{\circ}$
$40^{\circ}$
$20^{\circ}$
$0^{\circ}$ \begin{tabular}{|}
\hline \\
\hline \\
\hline
\end{tabular}

$b 6$

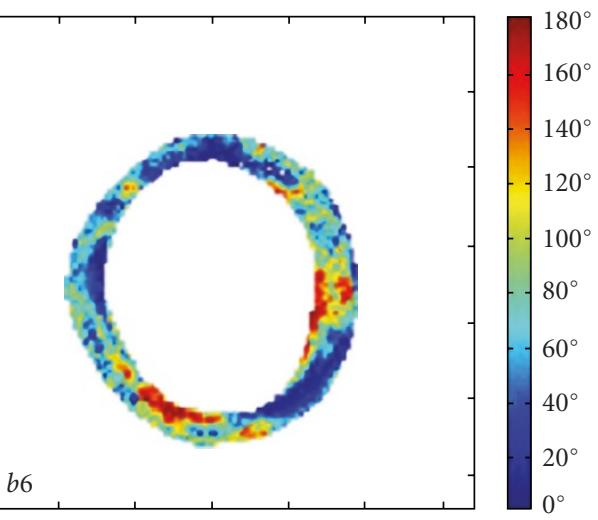

(f)

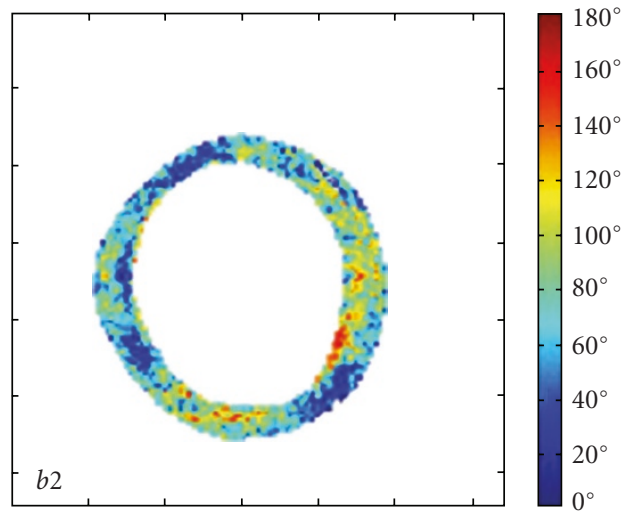

(b)

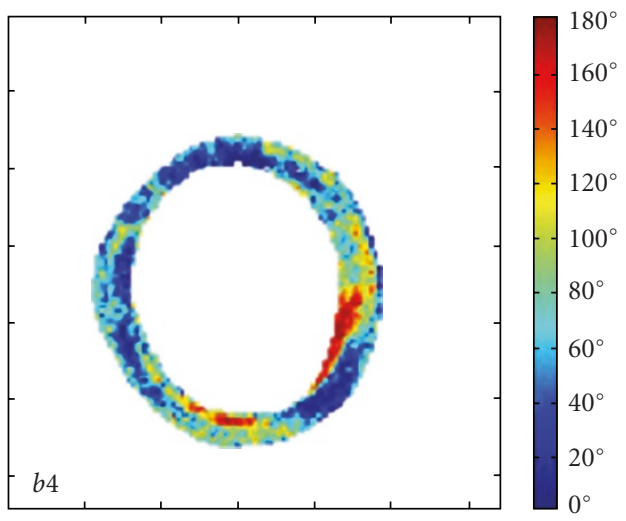

(d)

$0^{\circ}$

FIGURE 5: Contour maps of the angle between the first eigenvector and the $x-y$ plane for the averaged tensors of the image data sets for the different $b$ values.

tensor maps and the fibre tracts were analysed and data such as the eigenvector angle and fibre angle distribution were extrapolated. Finally, these data were compared over the different $b$ values in order to define the optimal one. It is possible to make this comparison by looking at the results shown in Figures 4-6.

For $b$ values less than or equal to $600 \mathrm{~s} / \mathrm{mm}^{2}$ it can be seen that while there is agreement with higher $b$ values in terms of the fibre angles plotted (Figures 6 and 7), the corresponding tensor map is not coherent. It can be seen in 


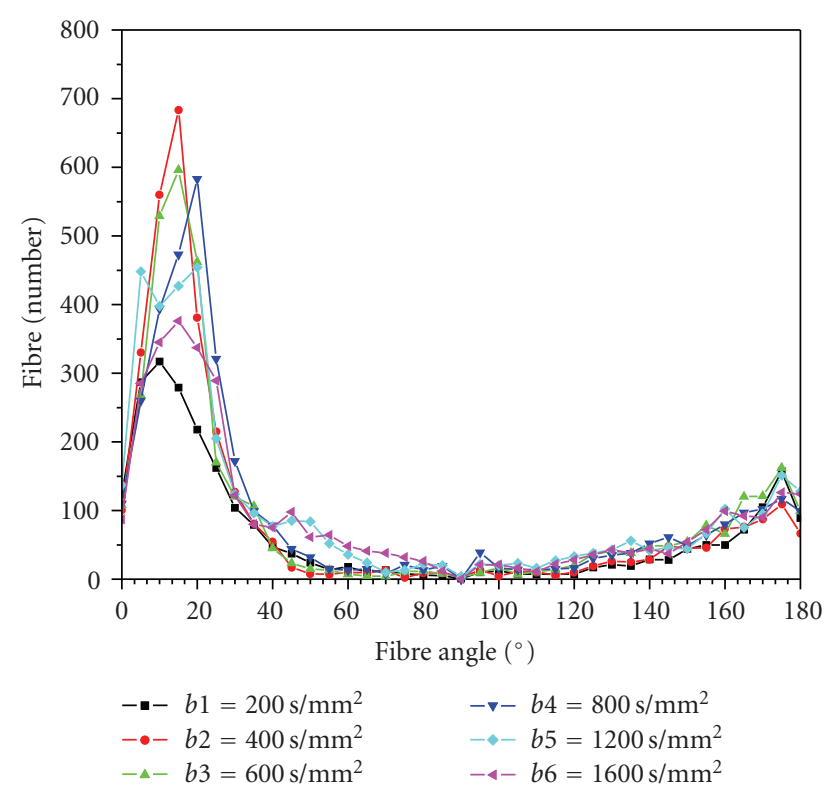

(a)

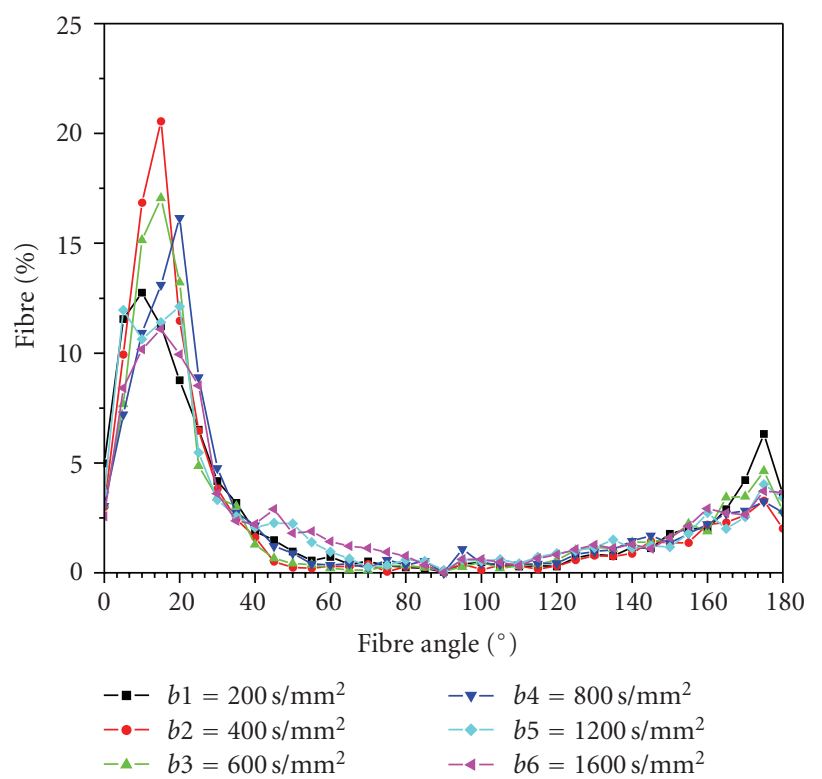

(b)

FIGURE 6: Distribution of the fibre angles over the analysed volume for different $b$ values, (a) number of fibres; (b) percentages of fibres. The fibre angles are evaluated over bands of $5^{\circ}$ and centered in the middle of each band.

At the same time, for $b$ values higher than $800 \mathrm{~s} / \mathrm{mm}^{2}$, the tensor maps show small changes, especially in Figure 5. This is confirmed in Figure 6 where the number of fibres with intermediate angles, especially in the range between $40^{\circ}-90^{\circ}$ obtained for $b 5$ and $b 6$ are higher than at $b 4$. In addition, the highest number of fibres is tracked for values in the range $b 2$ to $b 4$ whilst the number reduces from $b 2$ to $b 1$, and $b 4$ to $b 6$. These results suggest that the optimal $b$ value for arteries may be around $800 \mathrm{~s} / \mathrm{mm}^{2}(b 4)$, as this is the value

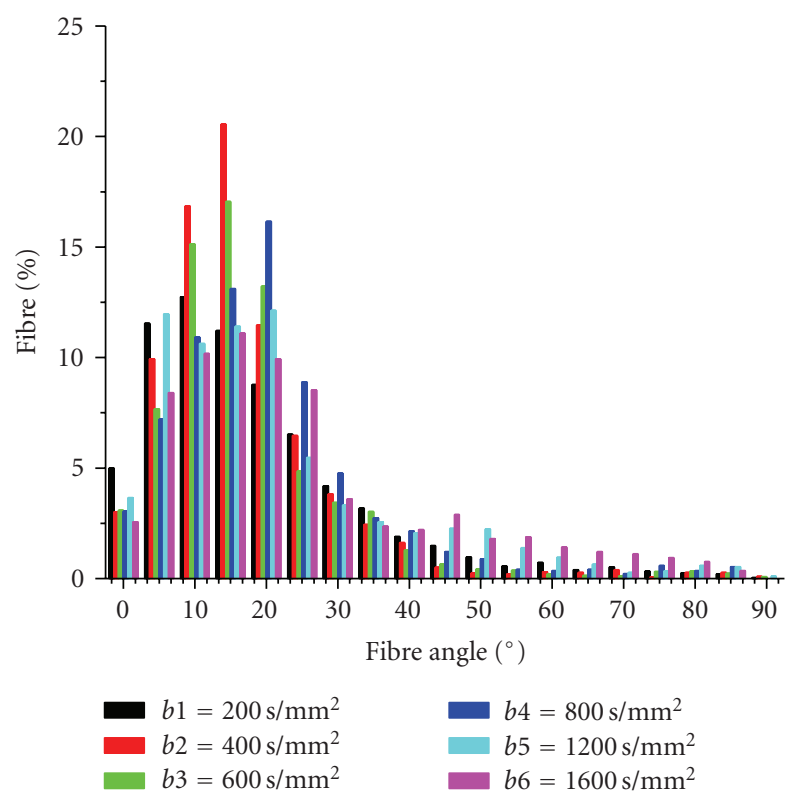

(a)

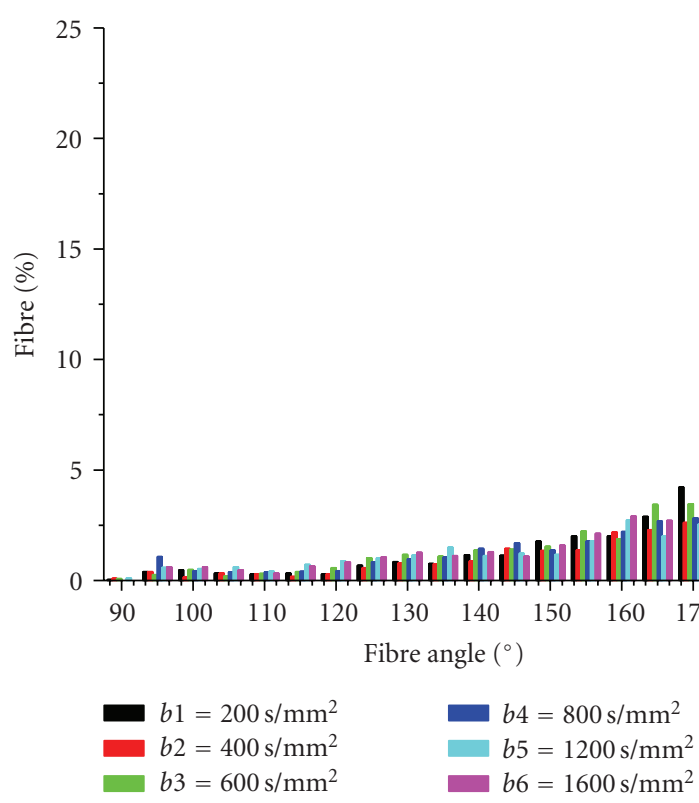

(b)

FIGURE 7: Histogram representing the fibre angle distribution for different $b$ values. For ease of representation it has been split into two graphs: (a) $0^{\circ}$ to $90^{\circ}$; (b) $90^{\circ}$ to $180^{\circ}$. The angles are evaluated over bands of $5^{\circ}$ and centered in the middle of each band.

for which there is a balance between the eigenvector angles in the tensor maps and the fibre data obtained by the fibre tracking procedure.

A limitation of this study is a lack of direct validation of these results through histology [27] or through other microscopic techniques [35-37]. The main objective of this study, however, was to use DTI for imaging the arterial structure and to compare the preliminary results obtained with the data available in the literature in order to show 
the feasibility of this approach. Another limitation was the analysis of a vessel in the unloaded configuration, whereby the circumferential stretch was preserved, but the longitudinal stretch was lost. Taking these limitations into account it is still clear that DTI of arterial tissue is feasible and that it can be used to successfully image the fibrous structure of arterial tissue in a noninvasive way.

In the future, DTI of an arterial vessel in a longitudinally tethered state and loaded with a pulsatile lumen pressure will be carried out, and finally the technique will be translated to an in vivo setting. The result of this procedure will be a noninvasive imaging technique with the potential to study the fibrous architecture of arteries in vivo which can be used for early diagnosis of arterial diseases.

\section{Appendix}

The appropriate tensor and tractography parameters were defined by means of a number of sensitivity tests. In order to obtain the most suitable value for each parameter, a range of values were applied and the differences observed between the results obtained considered, to establish the most suitable parameter for this particular application.

For the tensor parameters, the background suppression and the tensor smoothing had to be set. The background suppression consists in setting a threshold on the signal of the image under which no tensor will be estimated, based on the reference image $S_{0}$. This value was set to 1,000 (compared to a maximum signal of 32,766) in order not to interfere with the fibre tracking due to the fact that some areas of the arterial tissue were nearly as dark as the background. Regarding the tensor smoothing, a feature available in MedINRIA to reduce the noise of the tensor was set to "high," because by analysing the different FA maps, this was found to be the only value that would suppress the effect of the surrounding water.

For the fibre tractography, the optimal FA threshold, the minimum fibre length, the smoothness of the fibres, and the sampling parameters had to be established. The FA value was analysed first. Fractional anisotropy can be regarded as a tool to erase the noise and therefore the threshold value of FA influenced the amount of fibres tracked in the total image volume. The optimal FA should track fibres predominantly in the region of interest. For the aorta encased in fluid, extremely low FA threshold values $(<0.15)$ resulted in large amounts of fibres being tracked in the water volume, while high FA threshold values $(>0.3)$ resulted in very few fibres being tracked even in the aortic volume. Empirically we found that for an FA threshold value of 0.2 most of the fibres were tracked in the aortic volume and almost none in the water. Following the establishment of a suitable FA value, the effect of the minimum length of the fibre tracked was considered. Over two different sets of images, three different fibre lengths were considered; 5 , 10 , and $15 \mathrm{~mm}$. For a setting of $5 \mathrm{~mm}$ many fragmented fibres were tracked while for $15 \mathrm{~mm}$ only a few fibres, albeit extremely long fibres, were tracked. By comparison to available histological data on aortic tissue, a minimum fibre length of $10 \mathrm{~mm}$ showed an optimal tradeoff between the number of fibres and their length for all the different $b$ values and enabled a suitable sample from which average fibre directions could be ascertained. The value of $10 \mathrm{~mm}$ corresponds to one third of the planar resolution of the image. The influence of the smoothness parameter on the fibres tracked was also addressed. This parameter defines the smoothness of the curvature of the final fibre and ensures that large discontinuities in curvature from pixel to pixel of the image are identified such that they cannot be considered one fibre. For the chosen fibre length of $10 \mathrm{~mm}$, variations in the smoothness value over a large range $(20 \%-$ $80 \%$ ) showed that the fibre number tracked is relatively insensitive to this parameter and a value of $20 \%$ was applied to all subsequent images. Finally, the sampling parameter was investigated. The sampling parameter accounts for the number of pixels used for the determination of the fibre. A sampling parameter of one pixel, although extremely accurate, is computationally expensive. Empirically we found that a sampling parameter of three, where fibre tracking is only performed in one voxel out of each three, yields very good accuracy by comparison to a sampling of one pixel and reduces the computational time considerably [32].

\section{List of Symbols}

$S_{0}$ : Image with a null gradient (reference image)

$S$ : Image corresponding to an encoding gradient

$b i$ : Sensitivity to diffusion factor, $i=[1-6]$

$D$ : Diffusion tensor

$\lambda_{i}$ : Eigenvalues, $i=[1,2,3]$

$t$ : Angle with the $x$ axis

c: Helix lead constant

$R$ : Helix radius

$\theta$ : Fibre angle.

\section{Acknowledgment}

This project is funded by a Research Frontiers Grant (06/ RF/ENM076) awarded by Science Foundation Ireland.

\section{References}

[1] S. Petersen, V. Peto, M. Rayner, J. Leal, R. Luengo-Fernandez, and A. Gray, European Cardiovascular Disease Statistics, Department of Public Health, University of Oxford, Oxford, UK, 2005.

[2] M. A. Crowther, "Pathogenesis of atherosclerosis," in $A S H$ Education Program Book, pp. 436-441, American Society of Hematology, 2005.

[3] N. Sakalihasan, R. Limet, and O. D. Defawe, "Abdominal aortic aneurysm," The Lancet, vol. 365, no. 9470, pp. 1577-1589, 2005.

[4] G. Pasterkamp, D. P. V. de Kleijn, and C. Borst, "Arterial remodeling in atherosclerosis, restenosis and after alteration of blood flow: potential mechanisms and clinical implications," Cardiovascular Research, vol. 45, no. 4, pp. 843-852, 2000.

[5] D. N. Ku, "Blood flow in arteries," Annual Review of Fluid Mechanics, vol. 29, pp. 399-434, 1997.

[6] A. C. Burton, "Physical principles of circulatory phenomena: the physical equilibria of the heart and blood vessels," 
in Handbook of Physiology, American Physiological Society, Bethesda, Md, USA, 1959.

[7] G. S. Kassab, "Biomechanics of the cardiovascular system: the aorta as an illustratory example," Journal of the Royal Society Interface, vol. 3, no. 11, pp. 719-740, 2006.

[8] R. A. Peattie, T. J. Riehle, and E. I. Bluth, "Pulsatile flow in fusiform models of abdominal aortic aneurysms: flow fields, velocity patterns and flow-induced wall stresses," Journal of Biomechanical Engineering, vol. 126, no. 4, pp. 438-446, 2004.

[9] L. de Figueiredo Borges, R. G. Jaldin, R. R. Dias, N. A. G. Stolf, J.-B. Michel, and P. S. Gutierrez, "Collagen is reduced and disrupted in human aneurysms and dissections of ascending aorta," Human Pathology, vol. 39, no. 3, pp. 437-443, 2008.

[10] G. G. Hartnell, "Imaging of aortic aneurysms and dissection: CT and MRI," Journal of Thoracic Imaging, vol. 16, no. 1, pp. 35-46, 2001.

[11] S. G. Ruehm, M. Goyen, J. Barkhausen, et al., "Rapid magnetic resonance angiography for detection of atherosclerosis," The Lancet, vol. 357, no. 9262, pp. 1086-1091, 2001.

[12] C. Blondel, R. Vaillant, F. Devernay, G. Malandain, and N. Ayache, "Automatic trinocular 3D reconstruction of coronary artery centerlines from rotational X-ray angiography," in Proceedings of the Computer Assisted Radiology and Surgery, pp. 1073-1078, 2002.

[13] J. Golzarian, S. Murgo, L. Dussaussois, et al., "Evaluation of abdominal aortic aneurysm after endoluminal treatment: comparison of color Doppler sonography with biphasic helical CT," American Journal of Roentgenology, vol. 178, no. 3, pp. 623-628, 2002.

[14] Z. A. Fayad, V. Fuster, K. Nikolaou, and C. Becker, "Computed tomography and magnetic resonance imaging for noninvasive coronary angiography and plaque imaging: current and potential future concepts," Circulation, vol. 106, no. 15, pp. 2026-2034, 2002.

[15] J.-F. Toussaint, G. M. LaMuraglia, J. F. Southern, V. Fuster, and H. L. Kantor, "Magnetic resonance images lipid, fibrous, calcified, hemorrhagic, and thrombotic components of human atherosclerosis in vivo," Circulation, vol. 94, no. 5, pp. 932 938, 1996.

[16] P. J. Basser and C. Pierpaoli, "Microstructural and physiological features of tissues elucidated by quantitative-diffusiontensor MRI," Journal of Magnetic Resonance, Series B, vol. 111, no. 3, pp. 209-219, 1996.

[17] P. J. Basser and D. K. Jones, "Diffusion-tensor MRI: theory, experimental design and data analysis-a technical review," NMR in Biomedicine, vol. 15, no. 7-8, pp. 456-467, 2002.

[18] P. B. Kingsley, "Introduction to diffusion tensor imaging mathematics-part I: tensors, rotations, and eigenvectors," Concepts in Magnetic Resonance Part A, vol. 28, no. 2, pp. 101122, 2006.

[19] S. Mori and P. C. M. Van Zijl, "Fiber tracking: principles and strategies-a technical review," NMR in Biomedicine, vol. 15, no. 7-8, pp. 468-480, 2002.

[20] S. Mori and J. Zhang, "Principles of diffusion tensor imaging and its applications to basic neuroscience research," Neuron, vol. 51, no. 5, pp. 527-539, 2006.

[21] T. G. Reese, R. M. Weisskoff, R. N. Smith, B. R. Rosen, R. E. Dinsmore, and V. J. Wedeen, "Imaging myocardial fiber architecture in vivo with magnetic resonance," Magnetic Resonance in Medicine, vol. 34, no. 6, pp. 786-791, 1995.

[22] G. D. Buckberg, A. Mahajan, B. Jung, M. Markl, J. Hennig, and M. Ballester-Rodes, "MRI myocardial motion and fiber tracking: a confirmation of knowledge from different imaging modalities," European Journal of Cardio-Thoracic Surgery, vol. 29, supplement 1, pp. S165-S177, 2006.

[23] D. A. Lansdown, Z. Ding, M. Wadington, J. L. Hornberger, and B. M. Damon, "Quantitative diffusion tensor MRI-based fiber tracking of human skeletal muscle," Journal of Applied Physiology, vol. 103, no. 2, pp. 673-681, 2007.

[24] L. Filidoro, O. Dietrich, J. Weber, et al., "High-resolution diffusion tensor imaging of human patellar cartilage: feasibility and preliminary findings," Magnetic Resonance in Medicine, vol. 53, no. 5, pp. 993-998, 2005.

[25] P. Julkunen, R. K. Korhonen, M. J. Nissi, and J. S. Jurvelin, "Mechanical characterization of articular cartilage by combining magnetic resonance imaging and finite-element analysisa potential functional imaging technique," Physics in Medicine and Biology, vol. 53, no. 9, pp. 2425-2438, 2008.

[26] S. Capuani, C. Rossi, M. Alesiani, and B. Maraviglia, "Diffusion tensor imaging to study anisotropy in a particular porous system: the trabecular bone network," Solid State Nuclear Magnetic Resonance, vol. 28, no. 2-4, pp. 266-272, 2005.

[27] J. A. G. Rhodin, "Architecture of the vessel wall," in Handbook of Physiology, American Physiological Society, Bethesda, Md, USA, 1980.

[28] G. Andreisek, L. M. White, A. Kassner, G. Tomlinson, and M. S. Sussman, "Diffusion tensor imaging and fiber tractography of the median nerve at $1.5 \mathrm{~T}$ : optimization of $b$ value," Skeletal Radiology, vol. 38, no. 1, pp. 51-59, 2009.

[29] P. Mukherjee, J. I. Berman, S. W. Chung, C. P. Hess, and R. G. Henry, "Diffusion tensor MR imaging and fiber tractography: theoretic underpinnings," American Journal of Neuroradiology, vol. 29, no. 4, pp. 632-641, 2008.

[30] P. B. Kingsley, "Introduction to diffusion tensor imaging mathematics_-part II: anisotropy, diffusion-weighting factors, and gradient encoding schemes," Concepts in Magnetic Resonance Part A, vol. 28, no. 2, pp. 123-154, 2006.

[31] P. Filiard, X. Pennec, V. Arsigny, and N. Ayache, "Clinical DT-MRI estimation, smoothing, and fiber tracking with logEuclidean metrics," IEEE Transactions on Medical Imaging, vol. 26, no. 11, pp. 1472-1482, 2007.

[32] P. Fillard, J. C. Souplet, and N. Toussaint, Medical Image Navigation and Research Tool by INRIA (MedINRIA), INRIA Sophia Antipolis, Sophia Antipolis, France, 2007.

[33] E. Kreyszig, Advanced Engineering Mathematics, John Wiley \& Sons, New York, NY, USA, 1999.

[34] L. H. Peterson, R. E. Jensen, and J. Parnell, "Mechanical properties of arteries in vivo," Circulation Research, vol. 8, no. 3, pp. 622-639, 1980.

[35] J. M. Clark and S. Glagov, "Transmural organization of the arterial media. The lamellar unit revisited," Arteriosclerosis, Thrombosis, and Vascular Biology, vol. 5, no. 1, pp. 19-34, 1985.

[36] P. Farand, A. Garon, and G. E. Plante, "Structure of large arteries: orientation of elastin in rabbit aortic internal elastic lamina and in the elastic lamellae of aortic media," Microvascular Research, vol. 73, no. 2, pp. 95-99, 2007.

[37] M. K. O’Connell, S. Murthy, S. Phan, et al., “The threedimensional micro- and nanostructure of the aortic medial lamellar unit measured using 3D confocal and electron microscopy imaging," Matrix Biology, vol. 27, no. 3, pp. 171$181,2008$.

[38] C. Khalil, C. Hancart, V. Le Thuc, C. Chantelot, D. Chechin, and A. Cotten, "Diffusion tensor imaging and tractography of the median nerve in carpal tunnel syndrome: preliminary results," European Radiology, vol. 18, no. 10, pp. 2283-2291, 2008. 
[39] J. Chen, S.-K. Song, W. Liu, et al., "Remodeling of cardiac fiber structure after infarction in rats quantified with diffusion tensor MRI," American Journal of Physiology, vol. 285, no. 3, pp. H946-H954, 2003.

[40] W.-Y. I. Tseng, V. J. Wedeen, T. G. Reese, R. N. Smith, and E. F. Halpern, "Diffusion tensor MRI of myocardial fibers and sheets: correspondence with visible cut-face texture," Journal of Magnetic Resonance Imaging, vol. 17, no. 1, pp. 31-42, 2003.

[41] G. Tang, Y. Liu, W. Li, J. Yao, B. Li, and P. Li, "Optimization of b value in diffusion-weighted MRI for the differential diagnosis of benign and malignant vertebral fractures," Skeletal Radiology, vol. 36, no. 11, pp. 1035-1041, 2007.

[42] H. S. Seo, K.-H. Chang, D. G. Na, B. J. Kwon, and D. H. Lee, "High b-value diffusion ( $\left.b=3000 \mathrm{~s} / \mathrm{mm}^{2}\right) \mathrm{MR}$ imaging in cerebral gliomas at $3 \mathrm{~T}$ : visual and quantitative comparisons with $b=1000 \mathrm{~s} / \mathrm{mm}^{2}$," American Journal of Neuroradiology, vol. 29 , no. 3, pp. 458-463, 2008. 